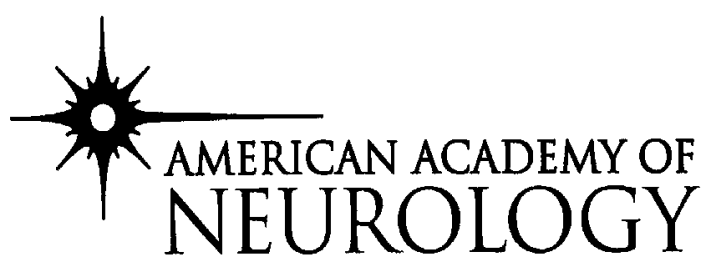

\title{
ASSESSMENT: NEUROLOGIC RISK OF IMMUNIZATION
}

\author{
Report of the Therapeutics and Technology Assessment Subcommittee \\ of the American Academy of Neurology
}

Gerald M. Fenichel, MD

Immunization programs are among the most cost-effective public health measures. They are the first line of defense against infectious disease, and when abandoned, epidemics often follow. Vaccines are biological products and some differences exist from lot to lot. Although no vaccine is $100 \%$ effective or safe, modern vaccines have an excellent safety record. Smallpox was eradicated by a global immunization program, and the eradication of poliomyelitis is within reach for the year 2000. Physicians are quick to blame vaccines for adverse neurologic events that follow immunizations. This bias probably originated with the first vaccines for rabies. They were grown in the CNS of mature animals, contained myelin basic protein, and often caused encephalomyelitis. In fact, no vaccine currently licensed for use in the United States is known to cause or exacerbate a demyelinating disorder of the CNS.

The United States maintains the most extensive obligatory childhood immunization schedule of any country, but has one of the worst records of infant immunization (table 1). The main reasons for the low immunization rate are deficiencies in health care delivery and access in the public sector. Full immunization is only realized at age 5, when it becomes prerequisite for school entry.

Immunization practices are recommended to the Surgeon General by the Advisory Committee on Immunization Practices (ACIP) of the Centers of Disease Control and Prevention. New recommendations of the ACIP are published in Morbidity and Mortality Weekly Reports and are the standard of care for immunization practice. The annual influenza vaccine is recommended by the ACIP for "any person who wishes to reduce the chance of becoming infected with influenza." One would think this should be everyone. Several vaccines are recommended for specific classes of adults: the aged and infirm, health care professionals, the military, and those traveling to countries where the risk of certain infectious diseases is high. Unfortunately, adults as a group are hesitant to accept immunization, and adult immunization rates are usually below needed levels. Neurologists have assumed the role of principal care providers for adults with certain neurologic disorders. As part of this role, they must recommend, and sometimes provide, vaccines for their patient

Table 1 Schedule of routine immunization of healthy infants and children Recommended

age Immunizations

\begin{tabular}{ll}
\hline Birth & HBV \\
2 mo & DTaP, HBV, HIB, OPV, or eIPV \\
4 mo & DTaP, HIB, OPV, or eIPV \\
6 mo & DTaP, HBV, HIB \\
$12-15$ mo & DTaP, HIB, MMR, OPV, or eIPV, Var \\
$4-6$ y & DTaP, MMR, OPV, or eIPV \\
$11-12$ y & DT, MMR, and Var if not given at or after 12 mo \\
\hline
\end{tabular}

From the American Academy of Neurology, St. Paul, MN.

Approved by the AAN Therapeutics and Technology Assessment Subcommittee October 3, 1998. Approved by the Practice Committee October 31, 1998. Approved by the Executive Board of the AAN February 27, 1999.

Received December 10, 1998. Accepted in final form January 2, 1999.

Address correspondence and reprint requests to TTA, American Academy of Neurology, 1080 Montreal Ave., St. Paul, MN 55116. 
$\mathrm{HBV}=$ hepatitis $\mathrm{B}$; DTaP = diphtheria, tetanus, and acellular pertussis; HIB = Haemophilus influenzae type b; OPV = oral poliomyelitis vaccine; eIPV = enhanced inactivated poliomyelitis vaccine; MMR = measles, mumps, rubella; Var $=$ varicella; DT $=$ diphtheria, tetanus.

Description of the analytic process. The National Childhood Vaccine Injury Act. Public Law 99-660 was passed by the US Congress in 1986. It established the Vaccine Injury Compensation Program (VICP) to evaluate claims of injuries from vaccines and to provide compensation when justified. The VICP is a no-fault system to compensate individuals who developed injuries lasting more than 6 months from vaccines that are universally recommended for children. Adults receiving covered vaccines, such as tetanus toxoid, or contracting paralytic poliomyelitis from someone who had been immunized with the oral vaccine, are also covered by the VICP. A table of injuries was established, outlining known injuries that are caused by covered vaccines, and the time frame in which such injuries should occur. Table injuries are presumed to be caused by the vaccine unless an alternate cause is established. The law required that the table of injuries be reviewed and updated regularly to reflect current scientific knowledge. The Institute of Medicine (IOM) was requested to undertake the first reviews and their reports were published in book form. ${ }^{1,2}$

These reports remain the best information available on what is known and not known concerning adverse events associated with routine childhood immunizations. The IOM took a novel approach in assessing the risks of individual vaccines because data concerning causality was often not available or inadequate. Rather than use the standard scientific approach that causality does not exist unless it is proved to exist, they reviewed the entire literature, considered biological plausibility, and categorized causality into the following five levels of proof:

1. There is no evidence bearing on a causal relationship.

2. The evidence is inadequate to accept or reject a causal relationship.

3. The evidence favors rejection of a causal relationship.

4. The evidence favors acceptance of a causal relationship

5. The evidence establishes a causal relationship.

Assessing causality. Adverse events associated with one vaccine should not be generalized to all other vaccines or even between different versions of the same vaccine. Neurologic disorders attributed to vaccine administration are generally the same as disorders that occur naturally. Therefore, when a neurologic event follows vaccine administration, it may not be possible for a physician to know whether the association is causal or coincidental. Unfortunately, much of the literature on vaccine-associated neurologic disorders was generated as case reports by individual physicians. Such reports often cause more concern than enlightenment, and cannot have scientific validity. The Vaccine Adverse Event Reporting System (VAERS) has been established as a national registry to collect information on possible vaccine-related injuries. Physicians who observe an adverse event in temporal relationship to vaccine administration are urged to report such incidents to VAERS and not to journals or public media. A VAERS reporting form is included in the back of the Physicians' Desk Reference.

Well-designed, randomized, controlled clinical trials, in which adverse events following immunization are compared in immunized and nonimmunized populations, are difficult and expensive to apply, and very few are available. Epidemiologic studies, using case-control or cohort methods, have been the main means to assess vaccine risk. Such studies can detect the relative risk of a commonly occurring adverse event but cannot detect the risk of a rare event (one in one million) unless it differs from naturally occurring diseases or has a biological marker. Epidemiologic studies rarely prove a cause-and-effect association. The quality of the evidence concerning adverse events associated with immunization in this report is rated according to the following system:

Class I: Evidence provided by one or more well-designed randomized controlled clinical trials

Class II: Evidence provided by one or more well-designed clinical studies such as case-control studies, cohort studies, etc.

Class III: Evidence provided by expert opinion, nonrandomized historical control subjects, or one or more case reports

Biological plausibility. Biological plausibility is an important consideration when assessing causation. Is the vaccine capable biologically of causing a specific adverse event? A possible confounding variable in assessing biological plausibility is antigenic mimicry wherein a peptide sequence in a vaccine component mimics a sequence of another protein such as myelin basic protein. This is a theoretical construct that has never been established as a cause of vaccine injury. 
To understand biological plausibility better, vaccines can be considered by their method of preparation (table

2). Four types of vaccines are available:

1. Vaccines composed of whole-killed organisms provoke an antibody response that provides temporary immunity. When prepared properly, they cannot produce the natural disease but could induce theoretically an immune-mediated or toxic reaction.

2. Live-attenuated virus vaccines are intended to cause an asymptomatic infection. However, properly constituted vaccines can cause symptomatic infection and the expected complications of the natural disease. The immunity provided by live-attenuated virus vaccines is similar to that conferred by the natural disease.

3. Vaccines can be composed of components of organisms or their denatured toxins (toxoids). This is only possible when the components that provide immunity or when the toxin that causes disease are known. Toxoids prevent disease but not infection. When prepared properly, they cannot produce the natural disease but could induce theoretically an immune-mediated reaction.

4. The hepatitis B vaccines are the only recombinant vaccines in general use. A portion of the hepatitis B virus gene coding for the surface antigen is cloned into yeast, and the vaccine is produced from cultures of the recombinant yeast strain. Vaccines prepared in this manner should have the lowest incidence of adverse reactions but could induce theoretically an immune-mediated reaction.

Table 2 Types of vaccines

\begin{tabular}{ll}
\hline Type & \multicolumn{1}{c}{ Vaccine } \\
\hline Whole-killed organisms & Influenza, pertussis, poliomyelitis (IPV), rabies \\
Live-attenuated viruses & Measles, mumps, poliomyelitis (OPV), rubella, varicella \\
Components of organisms & Acellular pertussis, Haemophilus infhienzae type B, diphtheria, tetanus \\
Recombinant & Hepatitis B \\
\hline IPV = inactivated poliomyelitis vaccine; OPV = oral poliomyelitis vaccine.
\end{tabular}

Summary of evidence. Diphtheria and tetanus toxoids. Adverse reactions to diphtheria toxoid in infants cannot be analyzed separately from tetanus toxoid because both are always administered together. No adverse events have been attributed to diphtheria toxoid. Tetanus toxoid is given alone after injury or burn exposure to children and adults. One unique patient developed three episodes of Guillain-Barré syndrome (GBS) after three doses of tetanus toxoid. The episodes were separated by 9 and 5 years, and the intervals between immunization and onset of symptoms were 3 weeks, 2 weeks, and 9 days. ${ }^{3}$ The patient subsequently experienced additional relapses without prior immunization and was diagnosed as having chronic inflammatory demyelinating polyneuropathy (CIDP). It is not possible to know whether the tetanus toxoid caused or triggered CIDP in a susceptible individual. A child was reported to the VICP who developed two episodes of GBS after tetanus toxoid administration. Although these are case reports (Class III), the IOM considered the association compelling for a cause-and-effect relationship because of the repeated response even though such an association cannot be established by epidemiologic studies (Class II).

Infants are reported who developed brachial neuritis after routine diphtheria, tetanus, and pertussis immunization. ${ }^{4}$ In such cases, the tetanus toxoid is implicated strongly even though these are only case reports (Class III). The IOM had concluded that a causal relationship exists between tetanus toxoid and brachial neuritis based on repeated reports in adults (Class III). Brachial neuritis does not occur spontaneously during infancy. All affected infants and most adults with brachial neuritis recover rapidly and completely.

Haemophilus influenzae type $\mathrm{b}$ (Hib). The original $H$. influenzae type b vaccine was a component vaccine consisting of the purified capsular polysaccharide polyribosylribitolphosphate (PRP). Because PRP vaccine was not effective in children younger than 18 months of age, the age when children are at greatest risk for H. influenzae meningitis, PRP was conjugated with other compounds to increase efficacy. PRP may be linked to the outer membrane protein complex of Neisseria meningitides (PRP-OMP), to diphtheria toxoid (PRP-D), to tetanus toxoid (PRP-T), or to a nontoxic mutant diphtheria toxin (HbOC). ${ }^{2,5}$ A few cases of GBS after Hib immunization have been reported, ${ }^{6,7}$ but the IOM concluded that "the evidence (Class III) is inadequate to accept or reject a causal relationship between Hib vaccines and GBS" (reference 2, page 246). Hib has been an extremely effective vaccine and has eliminated almost completely the threat of Haemophilus meningitis in the United States. ${ }^{8}$

Hepatitis B. A plasma-derived hepatitis B vaccine was used from 1982 to 1988; the recombinant product was initially used in late 1987 and has replaced the plasma-derived vaccine completely. Postmarketing surveillance for neurologic adverse events after the use of the plasma-derived vaccine showed a few cases of GBS, Bell's palsy, and 
brachial plexitis. ${ }^{9}$ A single case of acute cerebellar ataxia has been reported after the use of the recombinant vaccine. ${ }^{10}$ The evidence (Class III) is insufficient to support a cause-and-effect association. ${ }^{2}$

A more recent concern has been a reported association of hepatitis B vaccine and MS in adults but not children. ${ }^{11}$ The concern began when in 1991 hepatitis B immunization of health care workers became mandatory in France. Thousands of young and middle-age adults, mainly women, were immunized in a relatively short period of time. One neurologist saw several new cases of MS among such women and his experience was published in the public media (Class III). Epidemiologic evidence for a causal association has never been established. Several groups continue to study the issue. No reports have been published, but the incidence of either new cases of MS or exacerbations in established cases does not appear to be increased in recipients of hepatitis B vaccine.

Influenza. A new influenza vaccine is constituted each year depending on the prevalent viral strains that are expected to appear in the United States the following winter. Annual vaccination against influenza is recommended for everyone, but is especially needed for people with chronic diseases. A small increase in the incidence of GBS (slightly less than 10 patients per million persons vaccinated) was associated with the 1976 influenza vaccine (A, New Jersey-B, Victoria; Class II), and an increased risk of one to two per million doses has been identified with the influenza vaccines used in 1992 through 1994. During influenza epidemics from 1972 through 1995, estimated rates of influenza-associated death have ranged from approximately 300 to $>1,500$ per million persons aged 65 years and older. This age group accounts for more than $90 \%$ of all influenza-associated deaths. The potential benefits of influenza vaccination clearly outweigh the possible risks for vaccine-associated GBS. ${ }^{12}$

Neurologists have been hesitant to recommend the use of influenza vaceine in individuals with MS even though febrile illnesses, such as influenza, are known to cause relapse. It was their concern that the vaccine itself might cause relapse. A double-blind, placebo-controlled study of influenza yaccine given to patients with MS has shown that the vaccine does not induce relapse and should be used routinely in individuals with $\mathrm{MS}^{13}$ (Class I).

Measles, mumps, rubella (MMR). MMR are live-attenuated viral vaccines that are administered to infants as a combined vaccine. A live attenuated measles vaccine has been used in the United States since 1963; by 1982,97\% of all children were fully immunized by school entry against measles. The natural disease was eliminated in most states, and the incidence of measles had fallen from almost 500 per 100,000 in 1950 to 0.5 per 100,000 in 1982 . During the same period the total number of annual deaths from measles decreased from 700 to 2 , and reported cases of measles encephalitis decreased from 300 to $1 .^{14}$ Subacute sclerosing panencephalitis-a chronic form of measles encephalitis - has disappeared concomitantly. During the years 1989 through 1991, a resurgence of measles occurred among nonimmunized and not fully immunized individuals, causing 55,000 cases and 147 deaths. This resurgence serves as a reminder that waning disease is not absent disease.

The currently licensed measles vaceine uses the Edmonston B measles virus attenuated by prolonged passage in chick embryo cell culture. Children who receive live-attenuated measles vaccine may develop an asymptomatic case of measles. Some children develop fever, rash, and conjunctivitis in the second week after immunization (incubation period of at least 5 days). Theoretically, children with vaccine-induced measles could develop any of the known complications of natural infection. Conversely, adverse neurologic events that are not associated with natural measles infection are not caused by immunization. The main neurologic complication of measles immunization is febrile seizures in infants during the second week after immunization. ${ }^{15}$ Almost all children recover completely (Class II). However, a small number of cases of measles encephalitis with neurologic sequelae have been reported to the VICP. ${ }^{16}$ Although a cause-and-effect relationship has not been established, the relationship is biologically plausible (Class III).

The mumps vaccines, prepared in chick embryo cell culture, has eliminated natural mumps infection. No adverse neurologic events are associated with the mumps vaccine used in the United States, but a vaccine used in other countries, prepared from a different viral strain, has been associated with aseptic meningitis ${ }^{17}$ (Class III). Nine reports of sensorineural deafness after immunization with MMR have been reported (Class III). Three could be explained from other causes. The other six were unexplained, and if they were adverse events from MMR immunization, the mumps component would have the most biological plausibility. ${ }^{18}$

The present rubella vaccine is prepared from human diploid cells and produces an immune response that parallels the natural infection. The IOM concluded that the evidence is insufficient to indicate a causal relationship between the current rubella vaccine and radiculoneuritis and other neuropathies. ${ }^{1}$ However, the evidence is suggestive of a causal relationship between rubella vaccine and acute arthritis (Class III). As many as $40 \%$ of people receiving the current rubella vaccine may develop transitory arthralgias and paresthesias that begin 7 to 21 days after immunization and last from 1 to 3 days. These symptoms are mild and occur more often in adults than in children. Rubella virus vaccine is safe and effective. Neurologic complications have not been established. It has 
almost eradicated rubella embyropathy. Furthermore, rubella embyropathy has not occurred in children of women who were immunized inadvertently with rubella vaccine while pregnant.

Pertussis. Whole-cell pertussis vaccine has been used widely since 1947 to protect infants from this serious respiratory illness. Several neurologic disorders, especially seizures and encephalopathy, were attributed to the whole-cell vaccine (Class II). A causal relationship was never established for any complications except febrile seizures, unusual crying, and hypotonic-hyporesponsive episodes. A cause-and-effect relationship between wholecell pertussis vaccine and infantile spasms has been excluded specifically. The pathophysiology of hypotonichyporesponsive episodes is unknown; they are presumed to be syncopal and have no sequelae. The possibility that the whole-cell pertussis vaccine could cause an acute encephalopathy that resulted in a chronic brain damage syndrome was never fully excluded ${ }^{19}$ (Class II). No means is available by which a diagnosis of whole-cell pertussis vaccine encephalopathy can be established in an individual case.

An acellular vaccine was first recommended to replace the fourth and fifth doses of the whole-cell vaccine in 1992, and is now recommended for the primary immunizations as well. The vaccines licensed in the United States are combined with diphtheria and tetanus toxoids. Many acellular vaccines contain two or more denatured elements of Bordetella pertussis required for immunity but do not contain endotoxin, the substance responsible for local reactions and fever. The newest pertussis vaccine contains only one pertussis antigen - pertussis toxin. The incidence of unusual crying, hypotonic-hyporesponsive episodes, and seizures is reduced 10-fold using the acellular vaccine compared with the whole-cell vaccine ${ }^{20}$ (Class II).

The standard of practice concerning the administration of pertussis vaceine in infants and children with underlying neurologic disorders is stated in the Red Book, a report of the Infectious Disease Committee of the American Academy of Pediatrics. ${ }^{21}$ "The decision to give pertussis vaccine to infants and children with underlying neurologic disorders can be difficult and must be made on an individual basis after careful and continuing considerations of the risks and benefits" (reference 21, page 405). It is recommended that immunization with wholecell and acellular pertussis be deferred in children with progressive neurologic disorders. The concern is that administration of the vaccine "may coincide with or hasten the recognition of inevitable manifestations of the disorder, with resulting confusion about causation" (reference 21, page 405). It is also recommended to consider deferring immunization in infants and children who have either a personal history of seizures or known neurologic disorders that predispose to epilepsy. This recommendation is based on data that immunization with whole-cell pertussis increases the risk of seizures in such children. Similar data on acellular vaccines do not exist. Children with chronic brain damage disorders (cerebral palsy, epilepsy, and mental retardation) are often at greater risk from respiratory disorder, and I have recommended immunization with acellular vaccine.

Pertussis is a relatively common but often undiagnosed disorder in adults. Protection from childhood immunization is short lasting and adults are generally at risk for infection. In one prospective clinical study, ${ }^{22} 12 \%$ of adults who presented with cough lasting for 2 weeks or longer were shown to have pertussis infection as determined by ELISA immunoglobulin $\mathrm{G}$ antibody levels to pertussis toxin. Adult pertussis may be a debilitating disease, with cough lasting as long as 3 months. Ongoing studies of the acellular vaccines in adults are in progress. When the vaccine is licensed for adults, consideration should be given to the safety and feasibility of pertussis immunization in adults with chronic neurologic disorders.

Poliomyelitis. Inactivated poliomyelitis vaccine (IPV) was the first polio vaccine administered. In 1961, IPV was replaced in the United States by an orally administered live-attenuated viral vaccine (oral poliomyelitis vaccine [OPV]). The change occurred because OPV is administered more easily, confers humoral and mucosal immunity by infecting the gastrointestinal epithelial cells, and children immunized with OPV can spread the vaccine virus to nonimmunized persons and provide herd immunity. The disadvantage of OPV is that it can cause paralytic disease, whereas IPV causes only 2 days of low-grade fever in 5\% of recipients. All current cases of paralytic poliomyelitis in the United States are either vaccine-related or occurred in children who were exposed in other countries (Class II). The groups at risk are OPV recipients, nonimmunized contacts of OPV recipients, and immunodeficient individuals who were OPV recipients or contacts. ${ }^{23}$ The estimated overall frequency of paralytic disease in normal recipients or contacts is 1 per 2.5 million doses distributed. Approximately $93 \%$ of recipient cases and $76 \%$ of contact cases occur after the first two immunizations, $87 \%$ after the first. The interval between vaccine administration and onset of illness is 11 to 58 days. Healthy individuals tend to have a shorter latency than immunosuppressed individuals.

Ten patients with GBS were reported during an immunization campaign in Finland where more than one million doses of OPV were administered ${ }^{24}$ (Class III). Only five cases occurred within 3 weeks of immunization, and four occurred after 6 weeks. Two of the late cases occurred immediately after a diarrheal illness, suggesting the possibility of Campylobacter jejuni as the causative agent. The authors later reviewed the epidemic and found that 
the incidence of GBS had increased inexplicably in the months just before the OPV immunization program, and that the administration of OPV had not increased the incidence further ${ }^{25}$ (Class II). They concluded that the vaccine had not caused GBS.

An enhanced trivalent IPV (eIPV) has become the standard polio vaccine in Western Europe because it is safer than OPV and provides equivalent protection. The ACIP has recommended that the schedule of routine childhood immunizations be changed to encourage the expanded use of eIPV. ${ }^{26}$ eIPV may cause 2 days of fever but has no neurologic complications. eIPV will eventually replace OPV completely.

Rabies. The current rabies vaccine, prepared from rabies virus grown on human diploid cells (HDCV), has an excellent safety record. Rare cases of atypical GBS are reported after use of this vaccine, ${ }^{27}$ and one case of a seizure in temporal relationship to postexposure treatment has occurred ${ }^{28}$ (Class III). Although HDCV remains the gold standard, newer vaccines prepared on purified chick embryo cells appear to be equally effective both for pre- and postexposure rabies prophylaxis. ${ }^{29}$

Varicella. A live-attenuated varicella vaccine, first developed in 1974, is now available for routine childhood immunization. Its use is recommended for routine immunization in children. It is safe and effective in normal and immunocompromised children and has been shown to protect children with acute lymphocytic leukemia from natural varicella infection. ${ }^{30}$ The vaccine produces a mild case of chickenpox and may be followed by acute cerebellar ataxia (Class III). As in the cerebellitis following wild chickenpox infection, recovery is complete.

Table 3 Neurologic reactions to vaccines summarized

\begin{tabular}{llc}
\hline Vaccine & Reaction & Probability \\
\hline Measles & Febrile seizures & Consistently shown in population studies; \\
& Measles encephalitis & Rare, biologically plausible event; no \\
population studies
\end{tabular}

VICP $=$ Vaccine Injury Compensation Program.

Conclusions. Vaccines currently licensed for use in this country have an excellent safety record, and have greatly reduced the morbidity and mortality caused by infectious diseases in children and adults. All physicians should support the national vaccine initiative.

Recommendations. First, physicians should become better informed about the adverse reactions to modern vaccines and be hesitant to assume a cause-and-effect relationship based on only a temporal relationship. Also, physicians who observe an adverse event in temporal relationship to vaccine administration are urged to report such incidents to VAERS.

Disclaimer. This statement is provided as an educational service of the American Academy of Neurology. It is based on an assessment of current scientific and clinical information. It is not intended to include all possible proper methods of care for a particular neurologic problem or all legitimate criteria for choosing to use a specific procedure. Neither is it intended to exclude any reasonable alternative methodologies. The AAN recognizes that 
specific patient care decisions are the prerogative of the patient and thephysician caring for the patient, based on all of the circumstances involved.

\section{Acknowledgments}

The AAN TTA thanks Gerald Fenichel, MD, for his service to the Academy as the author of this assessment. The TTA also thanks the numerous individuals, AAN Sections, and organizations that reviewed the document, including the American Academy of Pediatrics, the Child Neurology Society, the Canadian Infectious Disease Society, and the American Public Health Association.

American Academy of Neurology Therapeutics and Technology Assessment Subcommittee members: John Ferguson, MD, Chair; Elliot Mark Frohman, MD, PhD; Robert Goldman, MD; Douglas Goodin, MD; Philip B. Gorelick, MD, MPH; Chung Hsu, MD, PhD; Andres Kanner, MD; Anne Marini, MD; E. Steven Roach, MD; Edward Westbrook, MD.

\section{References}

1. Institute of Medicine. Adverse effects of pertussis and rubella vaccines. Washington, DC: National Academy Press, 1991.

2. Institute of Medicine. Adverse events associated with childhood vaccines: evidence bearing on causality. Washington, DC: National Academy Press, 1993.

3. Pollard JD, Selby G. Relapsing neuropathy due to tetanus toxoid. J Neurol Sei 1978;37:113-125.

4. Hamati-Haddad A, Fenichel GM. Brachial neuritis following routine childhood immunization for diphtheria, tetanus, and pertussis (DTP): report of two cases and review of the literature. Pediatrics 1997;99:602-603.

5. American Academy of Pediatrics: Committee on Infectious Disease. Haemophilus type b conjugate vaccines: recommendations for immunization with recently and previously licensed vaccines. Pediatrics 1993;92:480488.

6. D'Cruz OF, Shapiro ED, Spiegelman KN, et al. Acute inflammatory demyelinating polyradiculopathy (Guillain-Barré syndrome) after immunization with Haemophilus influenzae type b conjugate. J Pediatr 1989;115:743-747.

7. Gervaix A, Caflisch M, Suter S, Haenggeli C-A. Guillain Barré syndrome following immunisation with Haemophilus influenzae type b conjugate vaccine. Eur J Pediatr 1993;152:613-614.

8. Murphy TV, White KE, Pastor P, et al. Declining incidence of Haemophilus influenzae type b disease since introduction of vaccination. JAMA 1993;269:246-248.

9. Shaw FE, Graham DJ, Guess HA, et al. Postmarketing surveillance for neurological adverse events reported after hepatitis B vaccination. Am J Epidemiol 1988;127:337-352.

10. Deisenhammer F, Pohl P, Bösch S, Schidauer C. Acute cerebellar ataxia after immunisation with recombinant hepatitis B vaccine. Acta Neurol Scand 1994;89:462-463.

11. Gout $\mathrm{O}$, Théodorou I, Liblau R, et al. Central nervous system demyelination after recombinant hepatitis B vaccination: report of 25 cases. Neurology 1997;48(suppl 3):A424. Abstract.

12. Centers for Disease Control and Prevention. Reports and Recommendations. MMWR 1998;42(RR-6):8-10.

13. Miller AE, Morgante E, Buchwald LY, et al. A multicenter, randomized, double-blind, placebo-controlled trial of influenza immunization in multiple sclerosis. Neurology 1997;48:312-314.

14. Bloch AB, Orenstein WA, Stetler HC, et al. Health impact of measles vaccination in the United States. Pediatrics 1985;6:524-532.

15. Griffin MR, Ray WA, Mortimer EA, Fenichel GM, Schaffner W. Risk of seizures after measles-mumps-rubella immunization. Pediatrics 1991;88:881-885.

16. Weibel RE, Caserta V, Benor D, Evans G. Acute encephalopathy followed by permanent brain injury or death associated with further attenuated measles vaccines: a review of claims submitted to the National Vaccine Injury Program. Pediatrics 1998;101:383-387.

17. Miller E, Goldacre M, Pugh S, et al. Risk of aseptic meningitis after measles, mumps, and rubella vaccine in UK children. Lancet 1993;341:979-982.

18. Stewart BJA, Prabhu PU. Reports of sensorineural deafness after measles, mumps, and rubella immunisation. Arch Dis Child 1993;69:153-154.

19. Institute of Medicine. DPT vaccine and chronic nervous system dysfunction: a new analysis. Washington, DC: National Academy Press, 1994. 
20. Centers for Disease Control. Pertussis vaccination: acellular pertussis vaccine for reinforcing and booster usesupplementary ACIP statement: recommendations of the Immunizations Practice Advisory Committee (ACIP). MMWR 1992;41(RR-1):1.

21. American Academy of Pediatrics. Pertussis. In: Peter G, ed. 1997. Red book: report of the Committee on Infectious Diseases. 24th ed. Elk Grove Village, IL: American Academy of Pediatrics, 1997:407-409.

22. Nennig ME, Shinefield HR, Edwards KM, Black SB, Fireman BH. Prevalence and incidence of adult pertussis in an urban population. JAMA 1996;275:1672-1674.

23. Strebel M, Sutter RW, Cochi S, et al. Epidemiology of poliomyelitis in the United States one decade after the last reported case of indigenous wild virus-associated disease. Clin Infect Dis 1992;14:568-579.

24. Kinnunen E, Färkkilä M, Hovi T, et al. Incidence of Guillain-Barré syndrome during a nationwide oral poliovirus vaccine campaign. Neurology 1989;39:1034-1036.

25. Rantala H, Cherry JD, Shields WD, Uhari M. Epidemiology of Guillain-Barré syndrome in children: relationship of oral polio vaccine administration to occurrence. J Pediatr 1994;124:220-223.

26. Institute of Medicine. Options for poliomyelitis vaccination inthe United States: workshop summary. Washington, DC: National Academy Press, 1996.

27. Bernard KW, Smith PW, Kader FJ, et al. Neuroparalytic illness and human diploid cell rabies vaccine. JAMA 1982;248:3136-3138.

28. Mortiere MD, Falcone AL. An acute neurologic syndrome temporally associated with postexposure treatment of rabies. Pediatrics 1997;100:720-721.

29. Dreesen DW. A global view of rabies vaccines for human use. Vaccine 1997;15(suppl):S2-S6.

30. White CJ, Kuter BJ, Hildebrand CS, et al. Varicella vaccine (Varivax) in healthy children and adolescents: results from clinical trials, 1987 to 1989. Pediatrics 1991;87:604-610. 


\title{
Neurology
}

\author{
Assessment: Neurologic risk of immunization [RETIRED]: Report of the Therapeutics and \\ Technology Assessment Subcommittee of the American Academy of Neurology \\ Gerald M. Fenichel \\ Neurology 1999;52;1546 \\ DOI 10.1212/WNL.52.8.1546
}

\section{This information is current as of May 1, 1999}

Updated Information \&

Services

Citations

Permissions \& Licensing

Reprints including high resolution figures, can be found at: http://n.neurology.org/content/52/8/1546.full

This article has been cited by 2 HighWire-hosted articles: http://n.neurology.org/content/52/8/1546.full\#\#otherarticles

Information about reproducing this article in parts (figures, tables) or in its entirety can be found online at:

http://www.neurology.org/about/about_the_journal\#permissions

Information about ordering reprints can be found online:

http://n.neurology.org/subscribers/advertise

Neurology ${ }^{\circledR}$ is the official journal of the American Academy of Neurology. Published continuously since 1951, it is now a weekly with 48 issues per year. Copyright . All rights reserved. Print ISSN: 0028-3878. Online ISSN:

$1526-632 X$.

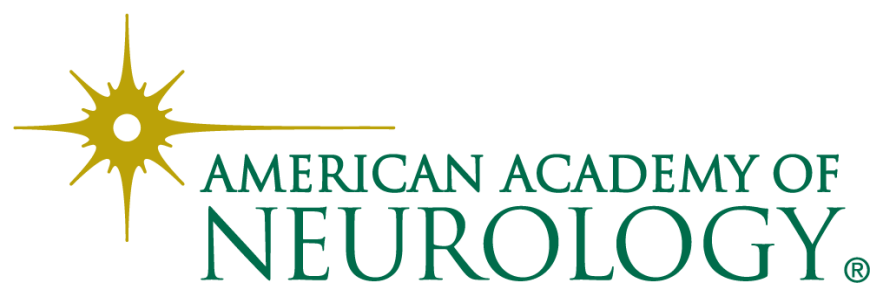

